\title{
Política pública de recursos humanos en la gestión de salud 2020
}

\author{
Loyda Marina Soto Cartel de Reyna \\ isotocastel@yahoo.es \\ Escuela de posgrado \\ Universidad César Vallejo \\ ORCID: 0000-0002-2216-5469
}

José Manuel Delgado Bardales jmdelgadob@ucvvirtual.edu.pe Escuela de posgrado Universidad César Vallejo ORCID:0000-0001-6574-2759 Scopus autor ID: 24070333700 Código Renacyt: P0050554

\section{RESUMEN}

La investigación tuvo como objetivo Caracterizar la política pública de recursos humanos 2020. La investigación fue básica de diseño descriptivo con revisión sistemática, las revisiones sistemáticas son investigaciones científicas en las cuales la unidad de análisis son los estudios originales primarios, constituyen una herramienta esencial para sintetizar la información científica disponible. Conclusiones, En función a los artículos científicos, sobre política de recursos humanos en salud pública evidencia que el 100\% corresponde a diferentes bases de datos, todos son estudios descriptivos con sus variantes análisis de revisión, análisis sistemático, casos y controles y estudio de casos. Las políticas públicas son las herramientas de gestión en toda institución pública, señala los procedimientos y actividades, siendo el medio por el cual el estado logra los objetivos planteados, por ende, las instituciones de salud deben adecuarse a la normativa establecida por el Estado. El recurso humano es el elemento fundamental para el logro de los objetivos institucionales y nacionales, siempre que esté satisfecho del trabajo que realiza y de las condiciones laborales que le favorecen. La evaluación permanente de las políticas públicas permitirá la toma de decisiones para el cambio de las situaciones problemáticas, generando retroalimentación y replanteamiento de las actividades estratégicas a fin de responder a los objetivos estratégicos planteados, siendo a su vez herramientas de evaluación para el cambio. Los países de las Américas muestran similitudes en el no avance de sus políticas públicas cuya funcionalidad está bajo la responsabilidad de cada nivel de gobierno.

Palabras clave: Política pública, recursos humanos, gestión en salud. 


\title{
Public policy of human resources in health management 2020
}

\begin{abstract}
The objective of the research was to characterize the public policy of human resources 2020. The research was basic of descriptive design with systematic review, systematic reviews are scientific investigations in which the unit of analysis are the original primary studies, they constitute an essential tool to synthesize the scientific information available. Conclusions, Based on the scientific articles on human resources policy in public health, it shows that $100 \%$ correspond to different databases, all are descriptive studies with their variants, review analysis, systematic analysis, cases and controls and case studies . Public policies are the management tools in every public institution, it indicates the procedures and activities, being the means by which the state achieves the proposed objectives, therefore, health institutions must adapt to the regulations established by the State. The human resource is the fundamental element for the achievement of the institutional and national objectives, provided that they are satisfied with the work they do and the working conditions that favor them. The permanent evaluation of public policies will allow decision-making to change problematic situations, generating feedback and rethinking strategic activities in order to respond to the strategic objectives set, being at the same time evaluation tools for change. The countries of the Americas show similarities in the failure to advance their public policies, the functionality of which is under the responsibility of each level of government.
\end{abstract}

Keywords: Public policy, human resources, health management.

Artículo recibido: 03 nov. 2020 Aceptado para publicación: 07 dic. 2020 Correspondencia isotore1476@ucvvirtual.edu.pe Conflictos de Interés: Ninguna que declarar 


\section{INTRODUCCIÓN}

Los Gobiernos que buscan el crecimiento económico, adoran invertir en capital físico, sin embargo, tienen menos interés en invertir en capital humano. Esto es un error, al descuidar las inversiones en capital humano, se puede debilitar drásticamente la competitividad de un país en un mundo en rápida transformación, en el que las economías necesitan cada vez más talento para sostener el crecimiento. (Banco Mundial, 2018).

Las estrategias empresariales sólo pueden realizarse a través de las personas. La única manera de mejorar el rendimiento empresarial es situar los recursos humanos en el centro mismo de la toma de decisiones estratégicas" (Conexiónesan, 2017).

El Plan Estratégico de la Organización Panamericana de la Salud (OPS) 2014-2019 destaca que el personal de salud es un actor político fundamental que tiene suficiente poder para cambiar la manera en que las políticas de salud se formulan y se ejecutan. La eficacia de la atención de salud depende enormemente del desempeño de los trabajadores de salud y, en consecuencia, de su financiamiento, capacitación, selección, contratación y desarrollo, y de ofrecerles perspectivas de carrera integrales. (Organización Panamericana de la Salud-OPS, 2014).

Destaca que los servicios de salud integrados de gran calidad, eficaces y centrados en la persona dependen de una combinación acertada de trabajadores de salud que tengan las aptitudes adecuadas, en el lugar adecuado y el momento propicio. El fortalecimiento de la gestión y el desarrollo de los recursos humanos para la salud deben formar parte de las políticas públicas. Puesto que los recursos humanos para la salud pueden influir considerablemente en el estado de salud de la población, deben considerarse como trabajadores esenciales y no como recursos flexibles que pueden recortarse fácilmente ante a un déficit presupuestario. OPS (2014).

En las Américas, las reformas del sector de la salud se enfrentan al desafío de fortalecer la función rectora de las autoridades sanitarias y una parte importante de este papel consiste en dar cumplimiento a las funciones esenciales de la salud pública (FESP) que competen al Estado en sus niveles central, intermedio y local. Para ello es crucial mejorar la práctica de la salud pública y los instrumentos para valorar su estado actual y las áreas en las que debe ser fortalecida.

En virtud de lo anterior, la Organización Panamericana de la Salud (OPS) ha puesto en marcha la iniciativa "La salud pública en las Américas", dirigida a la definición y medición de las FESP como base para mejorar la práctica de la salud pública y fortalecer el liderazgo de las autoridades sanitarias en todos los niveles del Estado. El presente artículo resume aspectos conceptuales y metodológicos relacionados con la definición y medición de las FESP y analiza las implicaciones de la medición del desempeño de las mismas en el mejoramiento de las prácticas de la salud pública en las Américas. Muñoz et al. (2000).

En el documento La Salud en las Américas 2002, se señala que los recursos humanos constituyen uno de los factores decisivos para provocar cambios en los sistemas de salud, acordes con las 
necesidades y demandas de la población; sin embargo, ha sido un aspecto al que las agendas políticas no le habían concedido la importancia que merece, pero que ha empezado a ganar prioridad. El mismo documento indica que los procesos de reforma han originado una superposición de problemas antiguos y nuevos. Entre los primeros se encuentran: la falta de equilibrio en la disponibilidad de personal; las inequidades en la distribución geográfica de los recursos; los desequilibrios en la composición del equipo de salud; el escaso desarrollo de los sistemas de información y la escasa integración entre formadores y empleadores. OPS (2003).

En la Evaluación de las Funciones Esenciales de Salud Pública (FESP) a nivel de la Autoridad Sanitaria Nacional Perú obtuvo un promedio del 63\%, correspondiente al nivel medio superior con una distribución asimétrica de las FESP, estos resultados corresponden a la segunda medición del desempeño luego de 5 años de haber definido la línea de base. Las FESP 5, 6 y 8 alcanzaron una calificación de 37\%, 30\% y 39\% respectivamente, considerado como desempeño medio inferior., siendo estas FEPS 5: Desarrollo de políticas y planes que apoyen los esfuerzos individuales y colectivos en salud pública y contribuyan a la rectoría sanitaria nacional. FESP 6: Fortalecimiento de la capacidad institucional de regulación y fiscalización en materia de salud pública. FESP 8: Desarrollo de recursos humanos y capacitación en salud pública. Ministerio de Salud (MINSA, 2006).

Por lo mencionado, se formuló el presente problema: ¿Cuáles son las características de la política pública de recursos humanos 2020?, y como Objetivo: Caracterizar la política pública de recursos humanos 2020.

\section{ESTRATEGIAS METODOLÓGICAS O MATERIALES Y MÉTODOS}

El tipo de investigación es básica de diseño no experimental descriptivo con revisión sistemática, las revisiones sistemáticas son resúmenes de investigaciones científicas disponibles en las que las unidades de análisis son los estudios originales primarios, se constituyen en una herramienta esencial para sintetizar la información científica disponible, incrementar la validez de las conclusiones de estudios individuales e identificar áreas de incertidumbre donde sea necesario realizar investigación (Hernández, 2016, p.217).

Es básico no experimental porque se recogió información tal y como se la encontró sin aplicar ningún programa durante el proceso, no se manipularon las variables y es descriptivo porque da a conocer las políticas públicas de recursos humanos utilizadas para producir mejoras en la gestión de salud (Tamayo y Tamayo, 2003. p.46). Por lo mencionado, se revisó información científica que mostro como la ejecución de las políticas públicas en recursos humanos pueden mejorar la gestión de salud.

La recolección de datos se realizó a través de una tabla de registro de datos y la fuente fue la revisión bibliográfica de artículos científicos nacionales como internacionales que tuvieron como tema las políticas públicas de recursos humanos y la gestión de salud, se seleccionaron los 10 artículos más 
importantes según el nivel de evidencia de los que se tuvo acceso al artículo completo de donde se recolectaron la información necesaria que permitieron el análisis.

La técnica de análisis se desarrolló a través de la revisión sistemática, analizando cada uno de los artículos seleccionados para establecer una comparación de los resultados y conclusiones que permita conocer cómo está el nivel de implementación y ejecución de las políticas públicas y su influencia en la gestión de salud, los avances de los mismos en el tiempo como las similitudes entre las políticas publicas. 


\section{RESULTADOS Y DISCUSIÓN:}

\section{Resultados}

Tabla 1: Estudios revisados sobre políticas públicas de recursos humanos en la gestión de salud 2020

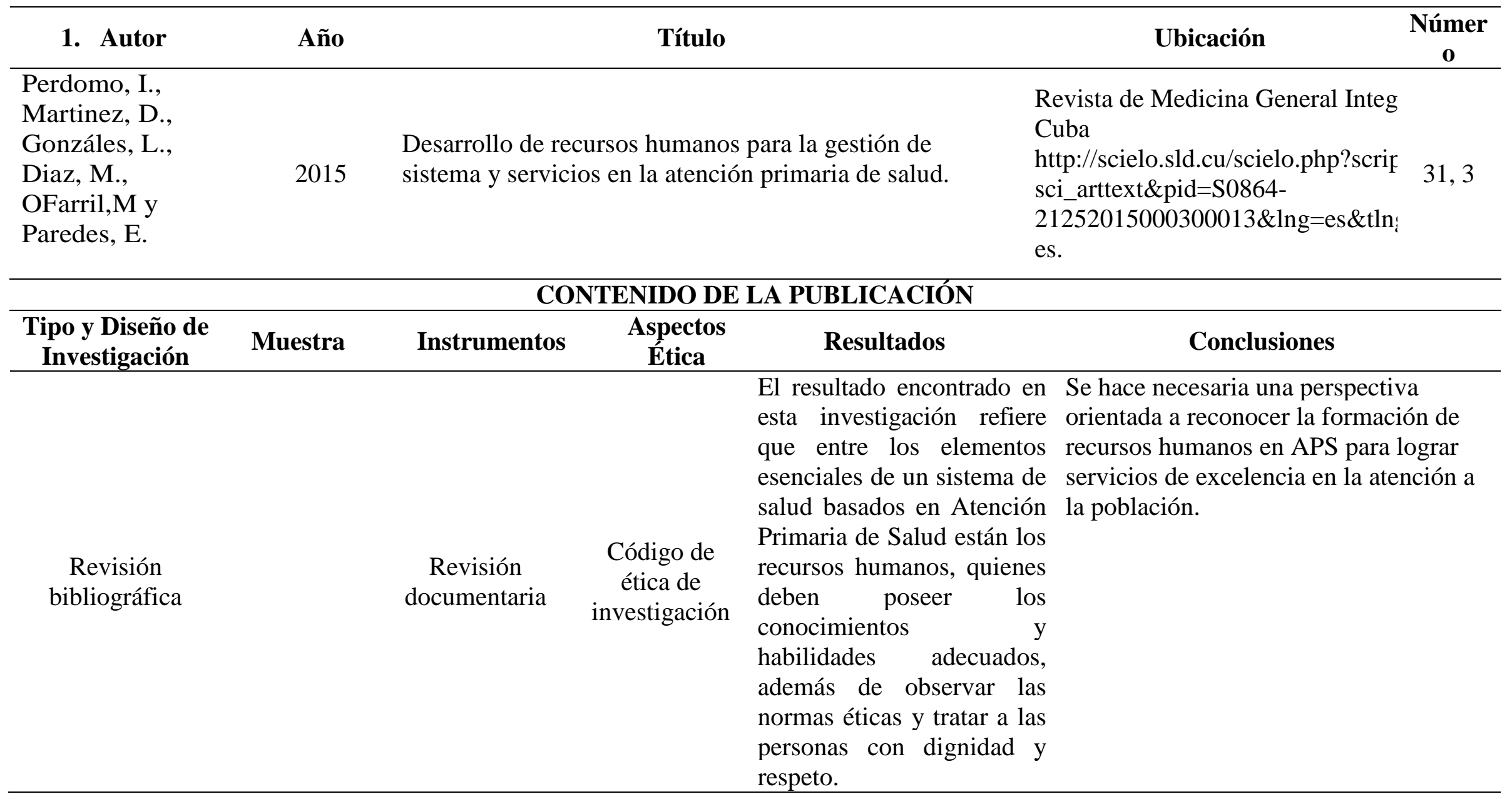




\begin{tabular}{|c|c|c|c|c|}
\hline 2. Autor & Año & Nombre de la Investigación & $\begin{array}{c}\text { Revista donde se ubica la } \\
\text { publicación }\end{array}$ & $\begin{array}{l}\text { Volúmen y } \\
\text { número }\end{array}$ \\
\hline $\begin{array}{l}\text { Buestos, V., Villarroel, } \\
\text { G., Ariza, F., Guillou, } \\
\text { M., Palacios,I., Cortez, } \\
\text { L., Cañizares, R., Núñez, } \\
\text { M y Huamán, L. }\end{array}$ & 2015 & $\begin{array}{l}\text { Análisis de la gestión de recursos humanos en los } \\
\text { modelos de salud familiar, comunitaria e intercultural de } \\
\text { los países andinos }\end{array}$ & $\begin{array}{l}\text { Anales de la Facultad de } \\
\text { Medicina. } \\
\text { Perú } \\
\text { https://dx.doi.org/10.15381/anal } \\
\text { es.v76i1.10971 }\end{array}$ & 76(spe), 49-5 \\
\hline
\end{tabular}

\section{CONTENIDO DE LA PUBLICACIÓN}

\begin{tabular}{|c|c|c|c|c|c|}
\hline $\begin{array}{l}\text { Tipo y Diseño de } \\
\text { Investigación }\end{array}$ & $\begin{array}{c}\text { Población y } \\
\text { muestra }\end{array}$ & Instrumentos & $\begin{array}{l}\text { Aspectos } \\
\text { ético }\end{array}$ & Resultados & Conclusiones \\
\hline $\begin{array}{l}\text { Estudio } \\
\text { cualitativo }\end{array}$ & $\begin{array}{c}\text { Gestores de } \\
\text { recursos } \\
\text { humanos. }\end{array}$ & $\begin{array}{c}\text { Diseño y } \\
\text { aplicación } \\
\text { de } \\
\text { instrumento }\end{array}$ & $\begin{array}{l}\text { Código de } \\
\text { ética en } \\
\text { investigación }\end{array}$ & $\begin{array}{l}\text { Los países han logrado avances en el } \\
\text { desarrollo de los sistemas de gestión } \\
\text { de recursos humanos de salud, } \\
\text { reconociéndose que los problemas } \\
\text { persistentes son la escasez e } \\
\text { inadecuada distribución del personal, } \\
\text { así como, perfiles profesionales de } \\
\text { egreso que no se ajustan plenamente a } \\
\text { las necesidades de salud derivadas del } \\
\text { cambio epidemiológico y de un } \\
\text { creciente compromiso por avanzar } \\
\text { hacia el acceso y cobertura } \\
\text { universales de salud. }\end{array}$ & $\begin{array}{l}\text { Estrategias innovadoras de } \\
\text { planificación, formación y gestión } \\
\text { del desempeño, podrían contribuir } \\
\text { a enfrentar los problemas de } \\
\text { recursos humanos de salud, en un } \\
\text { contexto adecuado de liderazgo y } \\
\text { gobernanza. }\end{array}$ \\
\hline
\end{tabular}




\begin{tabular}{|c|c|c|c|c|c|}
\hline Autor & Año & \multicolumn{2}{|c|}{ Nombre de la Investigación } & $\begin{array}{c}\text { Revista donde se ubica la } \\
\text { Publicación }\end{array}$ & Volumen Y Numero \\
\hline $\begin{array}{l}\text { García, H; Díaz, P; Ávila, } \\
\text { D y } \\
\text { Cuzco, M. }\end{array}$ & 2015 & \multicolumn{2}{|c|}{$\begin{array}{l}\text { La reforma del sector Salud y los } \\
\text { recursos humanos en salud }\end{array}$} & $\begin{array}{l}\text { Anales de la Facultad de } \\
\text { Medicina. } \\
\text { Perú } \\
\text { https://dx.doi.org/10.15381/anal } \\
\text { es.v76i1.10966 }\end{array}$ & 76(spe), 7-26 \\
\hline \multicolumn{6}{|c|}{ DESARROLLO } \\
\hline $\begin{array}{l}\text { Tipo y Diseño de } \\
\text { Investigación }\end{array}$ & $\begin{array}{l}\text { Población y } \\
\text { Muestra }\end{array}$ & Instrumentos & $\begin{array}{l}\text { Aspectos } \\
\text { Éticos }\end{array}$ & \multicolumn{2}{|c|}{ Conclusiones } \\
\hline $\begin{array}{l}\text { Revisión de bases de } \\
\text { información }\end{array}$ & $\begin{array}{l}\text { Revisión } \\
\text { documentaria }\end{array}$ & $\begin{array}{l}\text { Evaluación } \\
\text { avances }\end{array}$ & $\begin{array}{l}\text { Consentimiento } \\
\text { informado. }\end{array}$ & $\begin{array}{l}\text { Los recursos } \mathrm{h} \\
\text { claves para co } \\
\text { sistema nacio } \\
\text { hacia la atenci } \\
\text { reconocer el } \\
\text { todos. }\end{array}$ & $\begin{array}{l}\text { manos son las personas } \\
\text { struir e implementar el } \\
\text { al de salud orientado } \\
\text { n primaria de salud y a } \\
\text { arecho a la salud para }\end{array}$ \\
\hline
\end{tabular}




\begin{tabular}{|c|c|c|c|c|}
\hline Nombre & Año & \multicolumn{2}{|c|}{ Nombre } & Revista donde se ubica la Publicación \\
\hline $\begin{array}{l}\text { Zoio, G; } \\
\text { Cavada, A; } \\
\text { Sarmento, R y } \\
\text { Dal, M. }\end{array}$ & 2017 & \multicolumn{2}{|c|}{$\begin{array}{l}\text { Recursos humanos para la salud: } \\
\text { crisis global y cooperación } \\
\text { internacional }\end{array}$} & $\begin{array}{l}\text { Ciência \& Saúde Coletiva } \\
\text { Brasil } \\
\qquad \text { https://doi.org/10.1590/1413- } \\
81232017227.02702017\end{array}$ \\
\hline \multicolumn{5}{|c|}{ DESARROLLO } \\
\hline $\begin{array}{l}\text { Tipo y Diseño de } \\
\text { investigación }\end{array}$ & $\begin{array}{c}\text { Población y } \\
\text { Muestra }\end{array}$ & Instrumentos & $\begin{array}{l}\text { Aspectos } \\
\text { Éticos }\end{array}$ & Conclusiones \\
\hline $\begin{array}{l}\text { Revisión } \\
\text { narrativa } \\
\text { Revisión } \\
\text { documentaria } \\
\text { Descriptiva } \\
\text { longitudinal }\end{array}$ & & $\begin{array}{l}\text { Evaluación } \\
\quad \text { de } \\
\text { situacional. }\end{array}$ & $\begin{array}{l}\text { Código de ética } \\
\text { en } \\
\text { investigación }\end{array}$ & 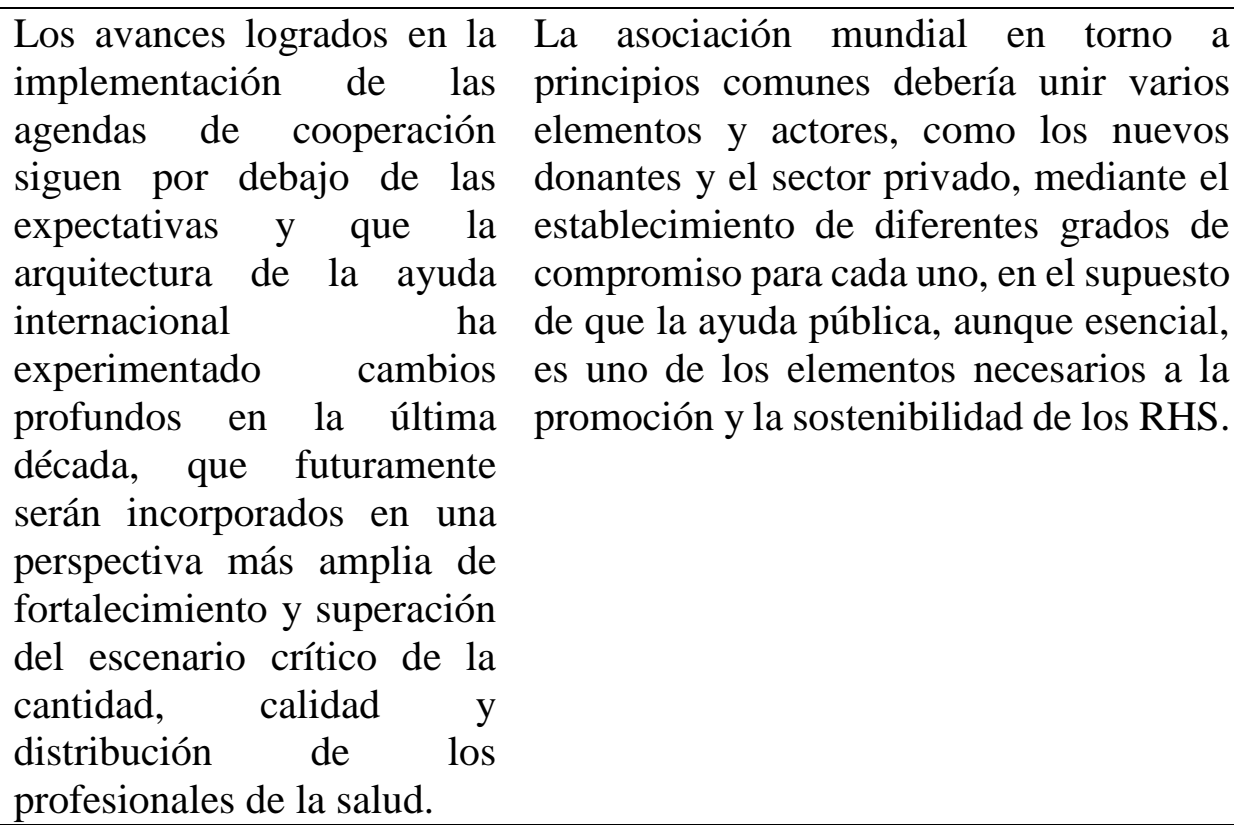 \\
\hline
\end{tabular}




\begin{tabular}{|c|c|c|c|c|}
\hline Autor & Año & Nombre de la Investigación & Revista donde se ubica la publicación & $\begin{array}{l}\text { Volumen y } \\
\text { número }\end{array}$ \\
\hline $\begin{array}{l}\text { Hernández, A y } \\
\text { Gómez, R. }\end{array}$ & 2015 & $\begin{array}{l}\text { Proceso de Gestión de Recursos } \\
\text { Humanos en un ámbito universitario }\end{array}$ & $\begin{array}{c}\text { Ingeniería Industrial/ISSN 1815- } \\
5936 \\
\text { Cuba } \\
\text { http://scielo.sld.cu/scielo.php?script= } \\
\text { i_arttext\&pid=S1815593620150002। } \\
\text { 007\&lng=es\&tlng=es. }\end{array}$ & $36-2$ p. $175-186$ \\
\hline
\end{tabular}

\section{DESARROLLO}

$\begin{array}{cccccc}\begin{array}{c}\text { Tipo y Diseño de } \\ \text { investigación }\end{array} & \begin{array}{c}\text { Población y } \\ \text { Muestra }\end{array} & \text { Instrumentos } & \begin{array}{c}\text { Aspectos } \\ \text { Éticos }\end{array} & \text { Resultados } & \text { Conclusiones }\end{array}$

Obtuvieron la descripción El diseño del proceso de Gestión de del proceso y de los 12 Recursos Humanos permitió definir de subprocesos. manera sistemática las actividades y tareas que componen a los subprocesos que lo conforman y definir las responsabilidades respecto al mismo. El uso del enfoque basado en procesos propició la

Lista de

Chequeo,

Diagrama

Revisión bibliográfica

Causa-Efecto,

Matriz DAFO

\section{Código de}

ética en

investigación identificación de los problemas que en concreto afectan esta área, así como la búsqueda de las soluciones concretas para atenderlos; sin que se afectaran el resto de los procesos de la organización. La identificación de las causas que originan las deficiencias en el proceso de Gestión de los Recursos Humanos, permite aplicar las medidas precisas para controlar $\mathrm{o}$ atenuar el efecto que originan en la eficiencia y eficacia del proceso. 


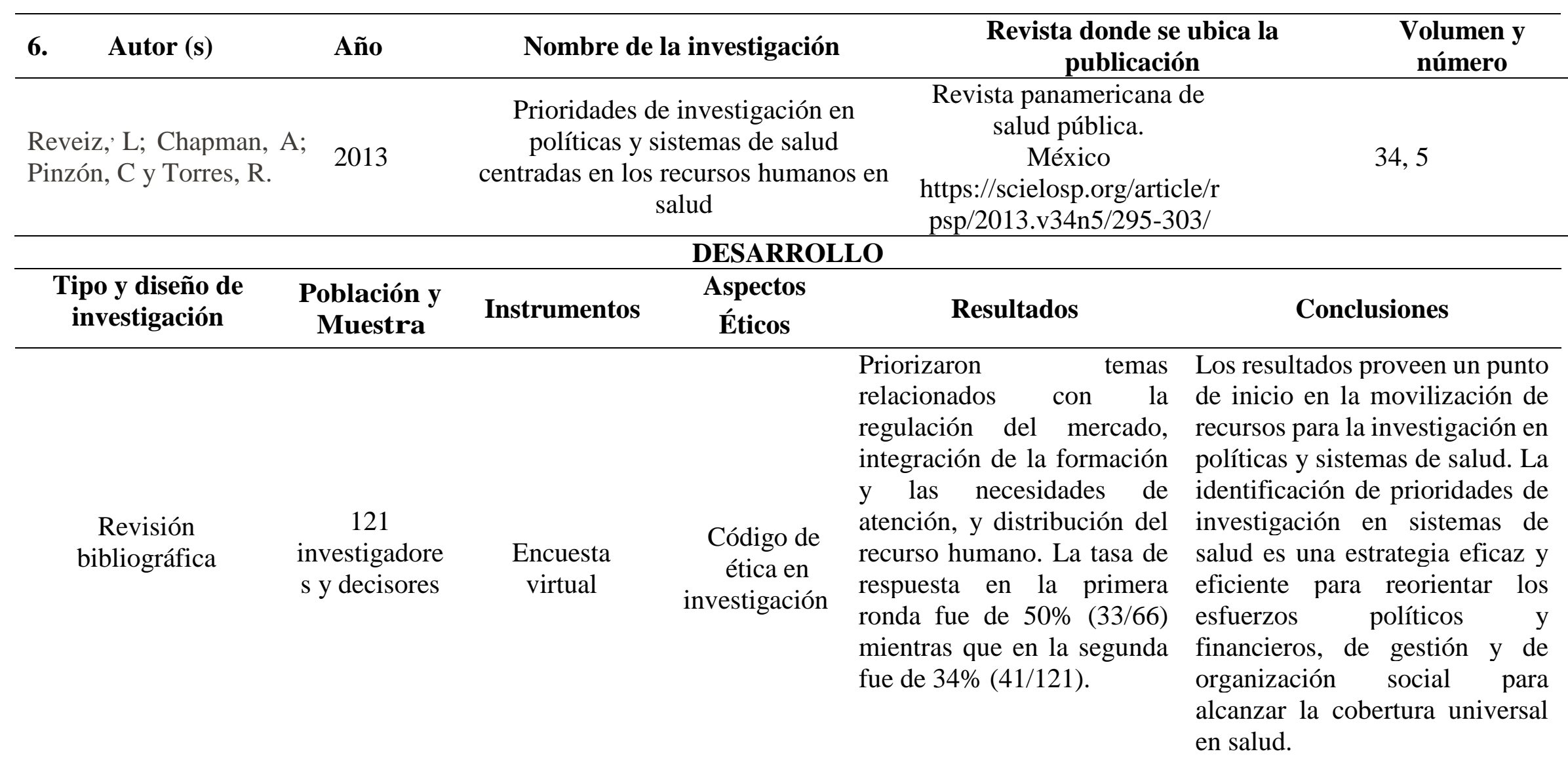




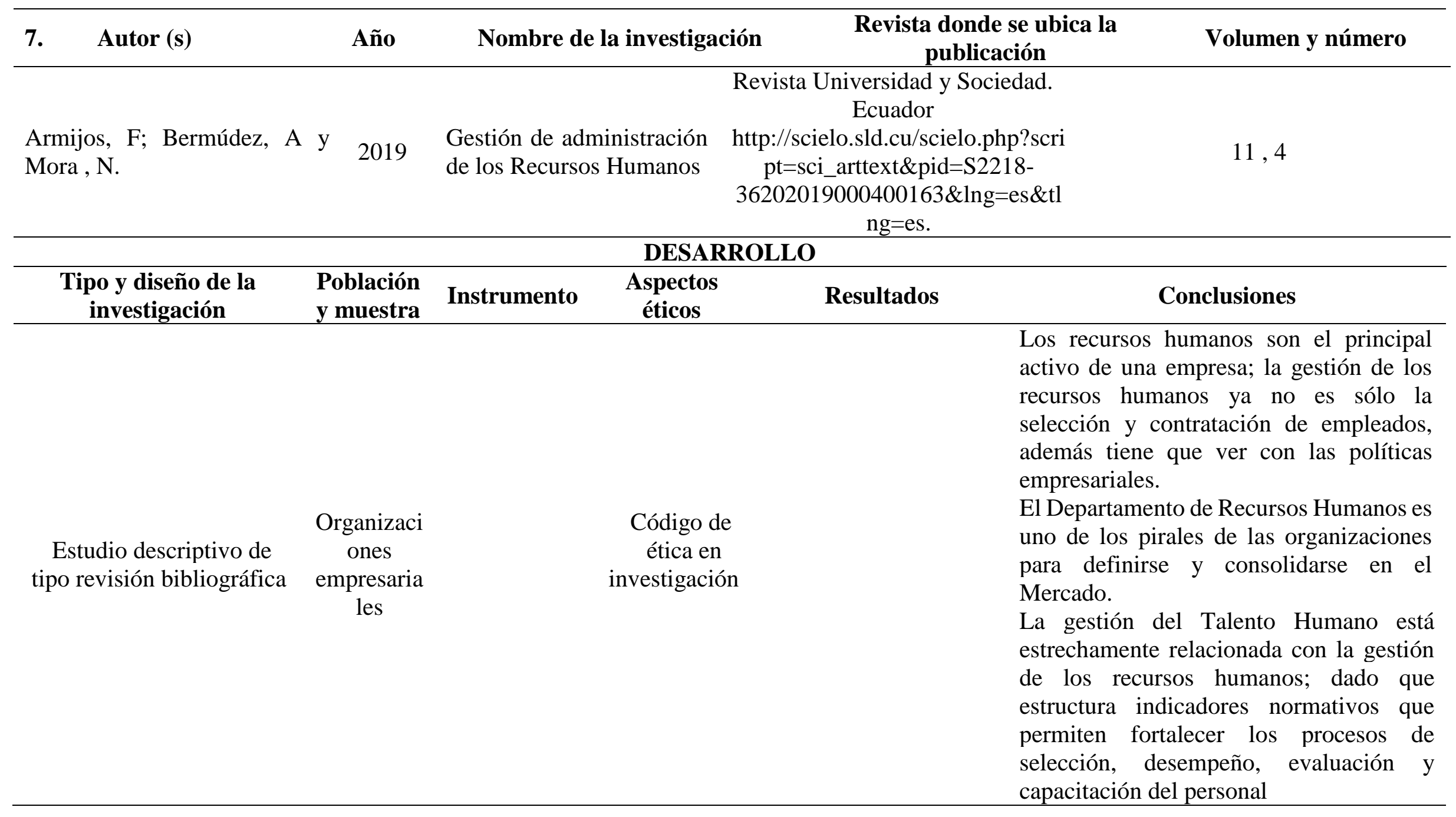




\begin{tabular}{|c|c|c|c|c|}
\hline Autor (s) & Año & \multicolumn{2}{|c|}{ Título } & Link y lugar \\
\hline Lobelle Fernández, Gretel & 2017 & \multicolumn{3}{|r|}{$\begin{array}{l}\text { Revista Alcance } \\
\text { Cuba } \\
\text { sld.cu/scielo.php?script=sci_art } \\
\text { text\&pid=S2411- } \\
000300006 \& \operatorname{lng}=\text { es\&tlng=es. }\end{array}$} \\
\hline \multicolumn{5}{|c|}{ DESARROLLO } \\
\hline $\begin{array}{c}\text { Tipo y diseño de la } \\
\text { investigación }\end{array}$ & $\begin{array}{l}\text { Población } \\
\text { y muestra }\end{array}$ & Instrumentos & Ética & Conclusiones \\
\hline Revisión bibiografica & & $\begin{array}{c}\text { Guías de } \\
\text { evaluación de } \\
\text { sistemas de } \\
\text { gestión como } \\
\text { soporte a } \\
\text { políticas } \\
\text { públicas } \\
\text { sociales. }\end{array}$ & $\begin{array}{c}\text { Código de } \\
\text { ética en } \\
\text { investigación }\end{array}$ & 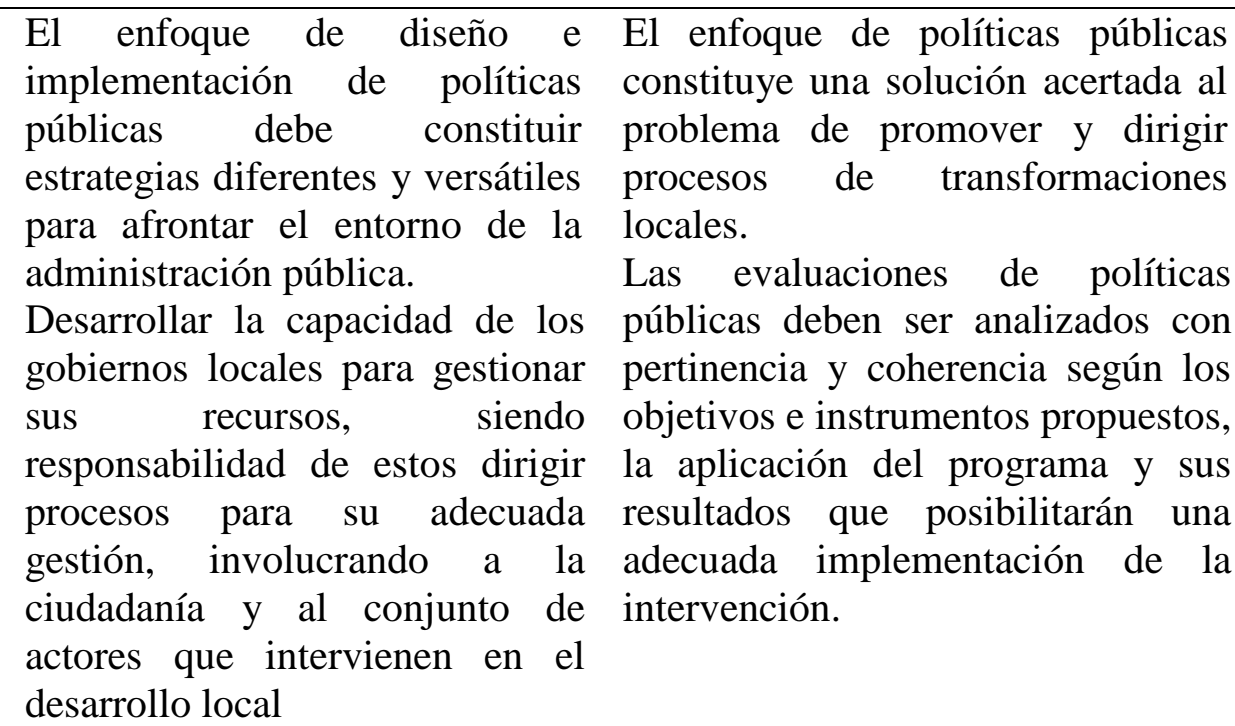 \\
\hline
\end{tabular}




\begin{tabular}{|c|c|c|c|c|c|}
\hline Autor (s) & Año & Títul & & $\begin{array}{c}\begin{array}{c}\text { Revista donde se ubica la } \\
\text { publicación }\end{array} \\
\end{array}$ & Volumen y número \\
\hline Rebolledo, Edgar. & 2015 & $\begin{array}{l}\text { Una política pública } \\
\text { del capital humano } \\
\text { municipales: aprendi } \\
\text { de la Academia } \\
\text { Municipal y Regiona }\end{array}$ & $\begin{array}{l}\text { ara el desarrollo } \\
\text { de funcionarios } \\
\text { ajes para el caso } \\
\text { Capacitación }\end{array}$ & $\begin{array}{c}\text { Rev. Est. de Políticas } \\
\text { Públicas. } \\
\text { Chile } \\
\text { http://dx.doi.org/10.5354/ } \\
\text { 0719-6296.2015.38429 }\end{array}$ & 1,2 \\
\hline \multicolumn{6}{|c|}{ DESARROLLO } \\
\hline $\begin{array}{l}\text { Tipo y Diseño de } \\
\text { Investigación }\end{array}$ & $\begin{array}{l}\text { Población } \\
\text { y Muestra }\end{array}$ & Instrumentos & $\begin{array}{l}\text { spectos } \\
\text { éticos }\end{array}$ & Resultados & Conclusión \\
\hline $\begin{array}{c}\text { Estudio de caso } \\
\text { Revisión bibliográfica }\end{array}$ & $\begin{array}{c}\text { 03 escuelas } \\
\text { de formación } \\
\text { de } \\
\text { funcionarios } \\
\text { públicos para } \\
\text { desarrollar el } \\
\text { capital } \\
\text { humano de } \\
\text { sus } \\
\text { respectivas } \\
\text { Administraci } \\
\text { ones } \\
\text { públicas. }\end{array}$ & $\begin{array}{c}\text { Normas } \\
\text { aplicable, } \\
\text { decretos, } \\
\text { estatutos, } \\
\text { memorias } \\
\text { anuales, } \\
\text { informes de } \\
\text { gestión, } \\
\text { comunicados } \\
\text { de prensa, } \\
\text { manuales de } \\
\text { operación, } \\
\text { lineamientos y } \\
\text { otros } \\
\text { documentos } \\
\text { disponibles en } \\
\text { los sitios web } \\
\text { de los casos } \\
\text { estudiados. }\end{array}$ & $\begin{array}{c}\text { Código de } \\
\text { ética en } \\
\text { investigación }\end{array}$ & $\begin{array}{l}\text { La Academia de } \\
\text { capacitación municipal y } \\
\text { regional de Chile cumple } \\
\text { un rol preponderante, como } \\
\text { aliada estratégica de los } \\
\text { municipios del país en } \\
\text { desarrollar el capital } \\
\text { humano calificado de los } \\
\text { funcionarios municipales. } \\
\text { Cuenta con una amplia } \\
\text { experiencia y camino } \\
\text { recorrido por } \text { otros } \\
\text { organismos públicos, tanto } \\
\text { nacionales } \\
\text { internacionales. }\end{array}$ & $\begin{array}{l}\text { El ejecutivo y el legislativo } \\
\text { muestran interés en } \\
\text { desarrollar el capital humano } \\
\text { calificado del sector } \\
\text { municipal. } \\
\text { Tiene la obligación de } \\
\text { convertirse en ra la } \\
\text { institucionalidad pública } \\
\text { que articule esfuerzos, } \\
\text { recursos, actores y } \\
\text { voluntades, a fin de que la } \\
\text { formación y capacitación } \\
\text { entregada este asociada al } \\
\text { ascenso, aumento de grados } \\
\text { y que los funcionarios que } \\
\text { esta forme y capacite sean } \\
\text { prioridad para el } \\
\text { reclutamiento y selección de } \\
\text { personas en cargos } \\
\text { disponibles en las } \\
\text { municipalidades } \\
\text { ingresen al sistema. }\end{array}$ \\
\hline
\end{tabular}




\begin{tabular}{|c|c|c|c|c|c|}
\hline Autor (s) & Año & \multicolumn{2}{|l|}{ Título } & \multicolumn{2}{|c|}{$\begin{array}{c}\text { Revista donde se ubica la } \\
\text { publicación }\end{array}$} \\
\hline $\begin{array}{l}\text { Molina, G; Oqu } \\
\text { Rodríguez, S; Mo } \\
\text { Vesga, C; La } \\
\text { Almanza, R; Cha } \\
\text { Goenaga, E y Arbo }\end{array}$ & $\begin{array}{l}\text { do, T; } \\
\text { oya, N; } \\
\text { s, N; } \\
\text { rro, M: } \\
\text { da,G. }\end{array}$ & $\begin{array}{l}\text { ón del talento hur } \\
\text { ca }\end{array}$ & no en salud & \multicolumn{2}{|c|}{$\begin{array}{l}\text { Revista Gerencia y politicas de } \\
\text { salud. } \\
\text { Colombia } \\
\text { https://doi.org/10.11144/Jave } \\
\text { riana.rgyps15-30.gtsp }\end{array}$} \\
\hline \multicolumn{6}{|c|}{ DESARROLLO } \\
\hline $\begin{array}{l}\text { Tipo y Diseño de } \\
\text { Investigación }\end{array}$ & $\begin{array}{l}\text { Población y } \\
\text { Muestra }\end{array}$ & imentos & $\begin{array}{l}\text { pectos } \\
\text { ticos }\end{array}$ & Resultados & Conclusión \\
\hline $\begin{array}{c}\text { Estudio } \\
\text { descriptivo de } \\
\text { corte } \\
\text { transversal }\end{array}$ & $\begin{array}{c}672 \text { personas } \\
\text { vinculadas a } \\
\text { instituciones de salud } \\
\text { habilitadas por el } \\
\text { Ministerio de Salud en } \\
\text { el 2013, que proveían } \\
\text { servicios y programas } \\
\text { de salud pública.. }\end{array}$ & $\begin{array}{l}\text { Un cuestionario } \\
\text { autodiligenciable, } \\
\text { cuyo contenido } \\
\text { incluye variables } \\
\text { sociodemográficas } \\
\text { (edad, sexo), } \\
\text { formación } \\
\text { académica, años } \\
\text { de experiencia, } \\
\text { tipo de contrato(s) } \\
\text { laboral(es) en los } \\
\text { últimos tres años } \\
(2012,2013 \text { y } \\
2014) .\end{array}$ & $\begin{array}{l}\text { Código de } \\
\text { ética en } \\
\text { investigación }\end{array}$ & $\begin{array}{l}\text { El } 26,6 \% \text { había realizado } \\
\text { capacitación por sus } \\
\text { propios medios. } \\
\text { El } 67 \% \text { de los encuestados } \\
\text { manifestó que en la } \\
\text { institución donde } \\
\text { laboraba se realizaban } \\
\text { procesos periódicos de } \\
\text { evaluación de personal } \\
\text { Un bajo porcentaje (23\%) } \\
\text { afirmó haber tenido algún } \\
\text { tipo de reconocimiento } \\
\text { por su trabajo. lo los } \\
\text { Las condiciones laborales } \\
\text { del personal de los } \\
\text { programas de salud } \\
\text { pública son } \\
\text { problemáticas. }\end{array}$ & $\begin{array}{l}\text { Limitada cobertura con los } \\
\text { programas capacitación en salud } \\
\text { pública ofrecidos por las } \\
\text { instituciones para las cuales } \\
\text { trabajan. } \\
\text { El salario, el reconocimiento y } \\
\text { el ambiente laboral fueron } \\
\text { ubicados como los aspectos que } \\
\text { más motivaban a las personas } \\
\text { encuestadas. } \\
\text { La idoneidad en salud pública } \\
\text { de las personas encuestadas se } \\
\text { asocia con la aprobación del } \\
\text { examen de conocimiento } \\
\text { durante el proceso de selección } \\
\text { para el cargo y con la existencia } \\
\text { de procesos de evaluación del } \\
\text { desempeño en las instituciones } \\
\text { donde los encuestados } \\
\text { trabajaban. }\end{array}$ \\
\hline
\end{tabular}




\section{DISCUSIÓN}

Posterior a la revisión sistemática de los artículos, encontramos que del 100\% corresponde a América latina y el Caribe, sobresaliendo los países de Cuba con 30\% y Perú con 20\%, seguido de los países de Brasil, México, Ecuador, Chile y Colombia con el 10\% respectivamente. En relación a los diseños y tipos de estudios el 100\% son descriptivos en sus diferentes variantes.

Después de analizar los resultados de los artículos seleccionados podemos concluir que las políticas públicas de recursos humanos son pilares fundamentales que guían la gestión de las instituciones, se comportan como herramientas que proveen al gestor los instrumentos que les permitirá realizar el proceso de planificación estratégica orientándose a los objetivos institucionales.

En virtud de lo señalado, el recurso humano adopta gran relevancia el mismo que es insustituible, por lo que los gobiernos muestran preocupación e interés en continuar estableciendo estrategias que permitan al capital humano contar con las herramientas que están señaladas en las políticas públicas de salud. Por su parte, Perdomo, I., Martinez, D., Gonzáles, L., Diaz, M., OFarril,M y Paredes, E. concluye que,. García, H; Díaz, P; Ávila, D y concluye que, es necesaria una perspectiva orientada a reconocer la formación de recursos humanos de forma integral y competente que permita atender adecuadamente a la población. También, Cuzco, M. determinó, que los recursos humanos son el elemento clave de los servicios por ello su buen estado laboral conlleva a la mejora de la calidad de la salud. Buestos, V., Villarroel, G., Ariza, F., Guillou, M., Palacios,I., Cortez, L., Cañizares, R., Núñez, M y Huamán, L, estableció que, se debe desarrollar estrategia innovadoras de planificación, formación y gestión para vencer los problemas de recursos humanos en salud, ello significaría que la integralidad de los colaboradores contribuye a mejora de los servicios sanitarios. Zoio, G; Cavada, A; Sarmento, R y Dal, M., concluye que, generar compromiso en el talento humano es indispensable para reducir efectos negativos en la atención de la salud, no debe generarse diferencias ente los servicios de salud públicos y privados.

Para Hernández, A y Gómez, R., concluyen que, amerita un proceso de gestión de recurso humanos que sistematice las actividades y tareas que componen a los subprocesos y definir 
responsabilidades respecto al mismo. Igualmente, Reveiz, L; Chapman, A; Pinzón, C y Torres, R., estableció que, que la movilización de recursos para la investigación en políticas y sistemas de salud es prioritaria para los recursos humanos, generando competencias investigativas que fortalezca sus competencias. Por otro lado, Armijos, F; Bermúdez, A y Mora, N., concluyeron que el principal activo de una empresa son el talento humano y debes er gestionado óptimamente en beneficio de la mayor captación de recursos.

Igualmente, Lobelle Fernández, Gretel, estableció que, el enfoque de políticas públicas constituye una solución acertada al problema de promover y dirigir procesos de transformaciones locales, incorporando evaluaciones de análisis pertinente y coherente. También, Rebolledo, Edgar, demuestran que el Estado tiene gran interés en fortalecer las capacidades del capital humano en el sector municipal enfocado a los equipos de la institucionalidad pública.

\section{CONCLUSIONES O CONSIDERACIONES FINALES}

En función a los artículos científicos, sobre política de recursos humanos en salud pública evidencia que el 100\% corresponde a diferentes bases de datos, todos son estudios descriptivos con sus variantes análisis de revisión, análisis sistemático, casos y controles y estudio de casos.

Las políticas públicas son las herramientas de gestión en toda institución pública, señala los procedimientos y actividades, siendo el medio por el cual el estado logra los objetivos planteados, por ende, las instituciones de salud deben adecuarse a la normativa establecida por el Estado.

El recurso humano es el elemento fundamental para el logro de los objetivos institucionales y nacionales, siempre que esté satisfecho del trabajo que realiza y de las condiciones laborales que le favorecen.

La evaluación permanente de las políticas públicas permitirá la toma de decisiones para el cambio de las situaciones problemáticas, generando retroalimentación y replanteamiento de las actividades estratégicas a fin de responder a los objetivos estratégicos planteados, siendo a su vez herramientas de evaluación para el cambio.

Los países de las Américas muestran similitudes en el no avance de sus políticas publicas cuya funcionalidad está bajo la responsabilidad de cada nivel de gobierno. 


\section{LISTA DE REFERENCIAS}

Armijos, F; Bermúdez, A y Mora, N. (2019). Gestión de administración de los Recursos Humanos. Revista Universidad y Sociedad. Ecuador. 11, 4 http://scielo.sld.cu/scielo.php?script=sci_arttext\&pid=S2218$36202019000400163 \& \operatorname{lng}=e s \& t \operatorname{lng}=e s$

Banco Mundial. (2018). Banco Mundial propone invertir en capital humano como vía al desarrollo. Mercado Global. Washington 27 junio, 2018. Disponible en: https://www.eldinero.com.do/62360/banco-mundial-propone-invertir-en-capitalhumano-como-via-al-desarrollo/

Buestos, V., Villarroel, G., Ariza, F., Guillou, M., Palacios,I., Cortez, L., Cañizares, R., Núñez, M y Huamán, L. (2015). Análisis de la gestión de recursos humanos en los modelos de salud familiar, comunitaria e intercultural de los países andinos. Anales de la Facultad de Medicina. 76(spe), 49-5. Perú. https://dx.doi.org/10.15381/anales.v76i1.10971

Conexionesan. (2017). ¿Qué es la estrategia empresarial y cómo ella se relaciona con el BSC?. Publicado el 19 de Julio 2017 a las 1:05 PM. https://www.esan.edu.pe/apuntes-empresariales/2017/07/que-es-la-estrategiaempresarial-y-como-ella-se-relaciona-con-el-bsc/

Fernando Muñoz, Daniel López-Acuña, Paul Halverson Carlyle Guerra de Macedo, Wade Hanna, Mónica Larrieu, Soledad Ubilla, y José Luis Zeballos. Las funciones esenciales de la salud pública: un tema emergente en las reformas del sector de la salud. Rev Panam Salud Publica/Pan Am J Public Health 8(1/2), 2000. https://scielosp.org/pdf/rpsp/2000.v8n1-2/126-134/es

García, H; Díaz, P; Ávila, D y Cuzco, M. (2015). La reforma del sector Salud y los recursos humanos en salud Anales de la Facultad de Medicina. Perú 76(spe), 7-26 https://dx.doi.org/10.15381/anales.v76i1.10966

Hernández, A y Gómez, R. (2015). Proceso de Gestión de Recursos Humanos en un ámbito universitario. Ingeniería Industrial/ISSN 1815-5936. 36 - 2 p. 175-186Cuba 
http://scielo.sld.cu/scielo.php?script=sci_arttext\&pid=S181559362015000200007 \&lng=es\&tlng=es

Hernández, Fernández y Baptista (2016). Metodología de la Investigación. Sexta edición, México. Recuperado de: http://observatorio.epacartagena.gov.co/wpcontent/uploads/2017/08/metodologia-de-la-investigación-sextaedicion.compressed.pdf

Lobelle Fernández, Gretel. (2017). Políticas públicas sociales: apuntes y reflexiones. $\begin{array}{llll}\text { Revista } & \text { Alcance } & \text { Cuba. } & \text { 34,5/295-303 }\end{array}$ http://scielo.sld.cu/scielo.php?script=sci_arttext\&pid=S241199702017000300006\&lng=es\&tlng=es

MINSA. Funciones esenciales en Salud Pública. DIGESA. Lima Perú. http://www.digesa.minsa.gob.pe/publicaciones/descargas/salud_americas/06-CH6--59-72.pdf

Molina, G; Oquendo, T; Rodríguez, S; Montoya, N; Vesga, C; Lagos, N; Almanza, R; Chavarro, M: Goenaga, E y Arboleda,G. (2016). Gestión del talento humano en salud pública. Revista Gerencia y políticas de salud. 15, 30. Colombia. https://doi.org/10.11144/Javeriana.rgyps15-30.gtsp

OPS / OMS. (2014). Plan estrategico 2014-2019. En Pro de la Salud: desarrollo sostenible y la equidad. Washington D.C. https://www.paho.org/hq/dmdocuments/2015/Plano-Estrat-2014-2019-ExecESP2.pdf?ua $=1$

OPS. (2003). La Salud en Las Américas. Revista Cubana Salud Pública 2003; 29(1):86-9. http://scielo.sld.cu/pdf/rcsp/v29n1/spu14103.pdf

Perdomo, I., Martinez, D., Gonzáles, L., Diaz, M., OFarril,M y Paredes, E. (2015). Desarrollo de recursos humanos para la gestión de sistema y servicios en la atención primaria de salud. Revista de Medicina General Integral. 31, 3. Cuba. http://scielo.sld.cu/scielo.php?script=sci_arttext\&pid=S0864$21252015000300013 \& \operatorname{lng}=$ es\&tlng=es

Rebolledo, Edgar. (2015). Una política pública para el desarrollo del capital humano de funcionarios municipales: aprendizajes para el caso de la Academia de Capacitaci'on 
Municipal y Regional. Rev. Est. de Políticas Públicas. 1,2. Chile. http://dx.doi.org/10.5354/0719-6296.2015.38429

Reveiz,, L; Chapman, A; Pinzón, C y Torres, R. (2013). Prioridades de investigación en políticas y sistemas de salud centradas en los recursos humanos en salud. 34, 5. Revista panamericana de salud pública. México. https://scielosp.org/article/rpsp/2013.v34n5/295-303/

Tamayo y Tamayo (2003). El proceso de la investigación científica. Cuarta edición. México. Editorial Limusa SA. ISBN 968-18-5872-75.4. https://books.google.com.mx/books?id=BhymmEqkkJwC\&printsec=frontcover\&hl $=\mathrm{es} \# \mathrm{v}=$ onepage $\& \mathrm{q} \& \mathrm{f}=$ false

Zoio, G; Cavada, A; Sarmento, R y Dal, M. (2017). Recursos humanos para la salud: crisis global y cooperación internacional. Ciência \& Saúde Coletiva. 22 (7), 2237-22 Brasil. https://doi.org/10.1590/1413-81232017227.02702017 